\title{
Comparison Studies of MANET-Satellite and MANET-Cellular Networks Integrations
}

\author{
Ye Miao, Zhili Sun, Ning Wang and Haitham Cruickshank
}

\begin{abstract}
A mobile ad hoc network (MANET) is a selfconfiguring infrastructure-less network. Taking advantage of spontaneous and infrastructure-less behavior, MANET can be integrated with satellite network to provide world-wide communication for emergency and disaster relieve services and can also be integrated with cellular network for mobile data offloading. To achieve different purposes, different architecture of integrated system, protocols and mechanisms are designed. For emergency services, ubiquitous and robust communications are of paramount importance. For mobile data offloading services, emphasis is amount of offloaded data, limited storage and energy of mobile devices. It is important to study the common features and distinguish of the architecture and service considerations for further research in the two integrated systems. In this paper, we study common issues and distinguish between two systems in terms of routing protocol, QoS provision, energy efficiency, privacy protection and resource management. The future research can benefit from taking advantage of the similarity of two systems and address the relevant issues.
\end{abstract}

Index Terms-MANET, integrated MANET-Satellite network, hybrid MANET-Cellular network

\section{INTRODUCTION}

A mobile ad hoc network (MANET) is a self-configuring infrastructure-less network with mobile devices connected by wireless. The advantage of this technology is that the communication devices can be inter-networked without any preexisting infrastructure (within their radio transmission range). Despite that MANETs are normally envisioned to operate as stand-alone networks, in order to accomplish specific tasks, they can be extended by integrating with other networks (e.g. satellite networks and cellular networks).

Due to the cost-effective and ease of deployment, MANETs are widely used in military services, emergency rescue operations and applications in infrastructure-less or some areas for extend communications. Satellite communication is considered to be crucial in the ubiquitous communication system as a result of its wide area coverage. In order to achieve a real sense of worldwide communication, satellite, sometimes, is the only solution for MANET to communicate with other parts of the world and bridge the digital divide [1][2]. Therefore, satellite is not only utilized as a component of an alternative routing path but also considered as part of integrated MANET-Satellite system.

With limited licensed spectrum available to cellular networks, tremendous growth of data traffic in recent years makes challenges for cellular networks to support the large volume of data. Data offloading is to use complementary network

Y.Miao, Z.Sun, N.Wang and H.Cruickshank are with the Institute for Communication Systems, University of Surrey, UK. E-mail: y.miao, z.sun, n.wang and h.cruickshank@surrey.ac.uk technologies and innovative techniques for diverting data from mobile/cellular networks in order to alleviate congestion and make better use of network resources. Working in unlicensed spectrum makes Wi-Fi a great candidate for data offloading. Smartphones, tablets and laptops are generating most of the mobile network traffic. Based on their geographically movement or their social behavior, these mobile devices can be formed as MANET. Under certain conditions, this network may be characterized by intermittent connectivity, long or variable delay, asymmetric data rate and high error rates (referred to opportunistic network). For the data which is not time-critical, the delivery can be achieved by the multiple hop networks [3][4]. This hybrid MANET-Cellular network enables intelligent data offloading and mobile nodes those have poor connections to cellular network leverage multi-hop routing to send/receive data.

Even though both systems benefit from ad-hoc network, they focus on different aspects and achieve their own service purposes. The main contribution of this paper is to provide comparison studies between integrated MANET-Satellite system for emergency services and hybrid MANET-Cellular network for data offloading. Based on the issues existing in MANET, we explore common issues and also distinguish challenges in different MANET based hybrid networks. This paper is organized as follows: in Section II, the architecture of integrated MANET-Satellite network is present. In Section III, the architecture of hybrid MANET-Cellular network for data offloading is described. We investigate the issues and challenges in two hybrid systems in Section IV. Finally, in Section V, we conclude the paper.

\section{SyStem ARCHITECTURE OF INTEGRATED SATELlite AND MANET FOR EMERGENCY SERVICES}

Among the different phases of public emergency situation management (preparedness, response, recovery, and mitigation), the adoption of a hybrid satellite terrestrial cooperative system turns out to be one of the most feasible solutions in the first-response phase of a disaster [5]. For the emergency services, the instant response and data reliability and the essential requirements for QoS. A number of programs have been investigated the integrated system in terms of various angles as is listed in Table I. Project MONET (Mechanisms for Optimization of hybrid ad-hoc networks and satellite networks) focuses on the architecture and routing protocols design, providing connectivity between nodes belonging to different ad-hoc networks [6]; project DUMBO (Digital Ubiquitous Mobile Broadband OLSR) emphasizes on delivery reliability of video streaming traffic and practical experiments 
are designed and carried out [7]; project SALICE (SatelliteAssisted Localization and Communication Systems for Emergency Services) focuses on the integration of communication and navigation allows the exploitation of both navigation information for communication purposes and communication support for navigation purposes [5].

The research studies show that satellite system plays the indispensable role for ubiquitous communication which leads to the question how. The first step is to design the system architecture. The authors in [8] pointed out that the satellite must be definitely looked as an integrated part of the global telecommunications infrastructure rather than as an individual entity and a possible integrated system concept was proposed. The system includes complete integration between terrestrial fixed networks, dynamic ad-hoc networks and satellite layer. As researches specifically for emergency response system have been actively carried out, the integrated system tends to be more effective and practical. A baseline scenario is depicted in Figure 1.

A MANET cluster consists of a set of MANET nodes connecting to each other by appropriate wireless links (e.g. WiFi). A general MANET node is equipped with WiFi interface and thus can connect to other node when they are in wireless link range. Each cluster has at least one node with satellite link capable of performing communications to a satellite. If the node is activated to communicate to satellite, it is called a gateway node. Some internetworking related operations are done in gateway nodes. The general node has all the services of a MANET node, including sending and receiving information without any specific task. A gateway node acts as interworking unit between the cluster and satellite network to reach the other clusters or networks. It is used to forward traffic for external network (e.g. another MANET or satellite network), which means it is possible to communicate with different types of networks. The only way for a mobile node to communicate with external networks is to communicate via a gateway node. Each MANET network has one or more gateway nodes capable of performing links to satellite network. When the node which has the potential to be a gateway is in de-active state, it is a potential gateway node. Under certain conditions, these nodes are activated to be gateway nodes to perform connections and allow MANET cluster to communicate with Network Control Center or other networks in remote area without direct link range and access information provided by other networks.

\section{SYSTEM ARCHITECTURE OF INTEGRATED MANET AND CELLULAR NETWORKS FOR DATA OFFLOADING}

Several research projects have been carried out to offload the mobile data to Wi-Fi access points (APs). However, in spite of the cost of configuration of APs, APs have a limited transmission range and do not offer a feasible solution for providing coverage of a large area. Hence, mobile devices based ad-hoc network is considered and designed to enlarge the data delivery range. Ad hoc mode has been used within mobilephone network to disseminate information and approved successful in some projects, e.g. Project Serval [9] and Project Dythr [10]. Project Dythr uses Wi-Fi ad hoc mode to disseminate local
TABLE I

FUNCTIONALITIES OF PROJECTS.

\begin{tabular}{l||c|c|c|c}
\hline Functionality & DUMBO & SatNEX & MONET & SALICE \\
\hline \hline $\begin{array}{l}\text { Terrestrial communica- } \\
\text { tion network integration }\end{array}$ & $\sqrt{ }$ & $\times$ & $\sqrt{ }$ & $\sqrt{ }$ \\
\hline $\begin{array}{l}\text { Satellite and terrestrial } \\
\text { network integration }\end{array}$ & $\sqrt{ }$ & $\sqrt{ }$ & $\sqrt{ }$ & $\sqrt{ }$ \\
\hline QoS optimization & $\sqrt{ }$ & $\times$ & $\sqrt{ }$ & $\sqrt{ }$ \\
\hline Security integration & $\times$ & $\sqrt{ }$ & $\sqrt{ }$ & $\times$ \\
\hline Energy efficiency & $\times$ & $\sqrt{ }$ & $\times$ & $\times$ \\
\hline Resource management & $\times$ & $\sqrt{ }$ & $\sqrt{ }$ & $\times$ \\
\hline Navigation integration & $\times$ & $\times$ & $\times$ & $\sqrt{ }$ \\
\hline
\end{tabular}

Fig. 1. System architecture of integrated MANET-Satellite network

headline messages to neighbors. The messages are included in service set identifier (SSID) which is an unique identifier attached to a specific wireless load area network (WLAN) and spread into network through Wi-Fi beacons. Mobile device can collect all the beacons it hears and forward its interested ones to others. Project Serval has developed mechanisms for mobile phones to keep communicating even when there is no network or it is not covered by the telecommunication infrastructures. The mobile phones involved created a Serval Mesh which is a completely functioned ad-hoc Wi-Fi network. They formed impromptu networks and smartphones are able to make calls and send messages without a mobile operator. Basically, it allows communication between mobile devices, essentially turning any device into a router temporarily, allowing the network to be formed and unfolding continuously, without user intervention. These studies show that mobile devices working on ad-hoc mode can be developed and used to forward and disseminate data successfully and efficiently. More advantage should be taken from this kind of application. Hence, a case of heterogeneous MANET-Cellular network has been proposed and studied [4] for data-offloading to alleviate the enormous data load in Cellular network. Most of the information delivered over mobile networks are multimedia newspapers, computer games, video clips, weather reports and so on. Benefiting from the nature of delay-tolerant of such applications, the information can be delivered to a small group of users and further disseminated to all mobile users. Mobile devices working in ad hoc mode act as part of this data offloading system. For example, some mobile users are subscribed to get certain content from mobile network. As shown in Figure 2, in order to reduce mobile data traffic, some non-real-time application data may be delivered to only a small fraction of selected users referring to target-users or helpers. Then target users will further disseminated the information among all the subscribed users through their 


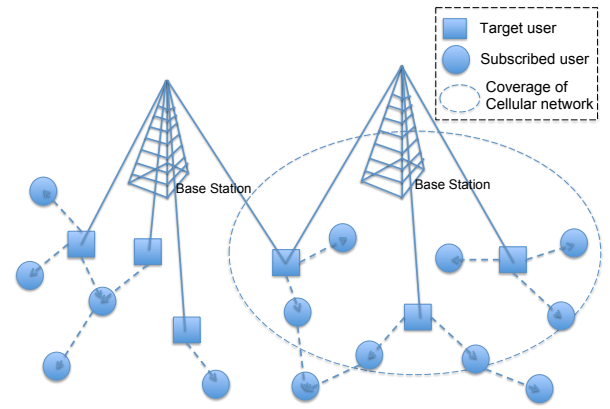

Fig. 2. System architecture of hybrid MANET-Cellular network.

networks where users can communicate with each other as along as they are within the transmission range. The target users and other subscribed users are able to connect and communicate with each other using Wi-Fi technology. The communication behavior of mobile devices can be described based on geographical topology or social behavior. Most of the study about ad-hoc based data offloading is designed for downlink traffic like in [11][12]. Even though the size of uplink traffic may not be as much as downlink, the number is much more larger and unpredictable. The architecture is also applicable to offload uplink traffic in a traffic constraint area to non-congested areas. For example, in [13], the authors focused on the spatial difference of the traffic among areas and balance the upload traffic of cellular networks.

Even through different terminology are used in MANETSatellite and MANET-Cellular system, we can see the similarity of the two integrated systems. Either satellite network or cellular network works as an external network provider. Mobile terminals/nodes are able to connect to each other locally in ad-hoc mode. To achieve different functionality, some of the mobile nodes have the access to external network and others don't. Thus, all the mobile nodes can exchange data with external network by ad-hoc communications and via the nodes with external network access. However, developed for different scenarios and user applications, the two integrated systems have their own considerations and challenges emphasizing on different aspects.

\section{Challenges AND OPEN ISSUES IN INTEGRATING MANET WITH OTHER NETWORKS}

As mentioned, benefitting from the spontaneous communication and other advantages, MANET can be integrated with satellite and cellular network to serve for different purposes. On the other hand, MANET can also suffer from limitations such as lack of central management and control. For integrated network, some features might be compromised and some might be amplified. In this section, we examine the challenges and issues of the two systems in terms of routing protocol, QoS optimization, energy efficiency mechanisms, privacy and security consideration. Figure 3 shows the design considerations of integrated network.

\section{A. Intermediary selection and Routing protocol}

In the integration system, the essential issue is to achieve the interoperability of two end networks. Thus, working for bridging and propagating traffic between two networks, the intermediary plays a significant role. Each system has its own term for the intermediary - gateways in MANET-Satellite system and target users in MANET-Cellular system. Given the application and end user forms, the routing about information dissemination can be different.

In integrated MANET-Satellite network, gateway selection is a key part of intelligent MANET routing. Undoubtedly, the priority job is to guarantee the end-to-end delivery of data packets. An efficient and intelligent routing protocol should also be able to provide flexible adjustments for variable network conditions. Hence, to deal with some unexpected situations (e.g. unreachability of gateway or failure of satellite link), efficient gateway handover mechanism is essential to be designed in the routing protocol. Project MONET [6] proposes a fault tolerant mechanism to mitigate the effect of path failure. Memorizing more than one possible neighbors, a single node is able to redirect the traffic through an alternative neighbor as soon as a link failure occurs. As part of routing protocol optimization, load balancing cannot be neglected. A better load-balance between different gateways alleviates congestion in an overloaded gateway as well as increases data rate on an underused gateway, in hence improving the overall throughput. In [6], they proposed a gateway selection method for end users to take into account gateway load balancing.

In the ad-hoc-based mobile data offloading system, to diffusion information which offloaded from cellular network, the selection of target users(also referred as helpers) are of key importance. Different from the gateway selection, the basic purpose in this system is to maximize the infected users with minimum resource consuming in terms of initial target users or energy. The authors in [11] considered a mobility-assisted wireless podcasting service to offload the operators network. In this system, the podcast contents are relayed from one user to another in a store-carry-forward way. In order to minimize the spectrum usage in cellular network, they simply select $p$ percentage of the subscribers with the strongest propagation channels as their target users. In [12], authors study how to choose the initial target set with just a certain number of users and maximize the expected number of infected users. Based on the mobile social network contact graph, they exploit a probabilistic dissemination model considering the realistic information dissemination duration. The authors in [13] investigate the data offloading for uplink traffic. In the area where traffic load is high, delay-tolerant data generated from a mobile device will be directly forwarded to a nearby device which is moving towards to a low traffic area. When the data is carried to other areas with low traffic load, it is forwarded to a cellular base station. Hence, the congestion of cellular base station in high density traffic area can be alleviated. They use particle filter to deduce a user behavior focusing on the spatial difference of a level of traffic load instead of predicting a user location.

To compare the two systems, given the desirable QoS requirements, taking advantage of the ad-hoc distributed feature and load-balancing is the common goal. In MANET-Satellite network, load-balancing is to make best use of both adhoc in-network and satellite resources. In MANET-Cellular network, load-balancing is to divert the mobile data from cellular network to local ad-hoc network. In general, the 


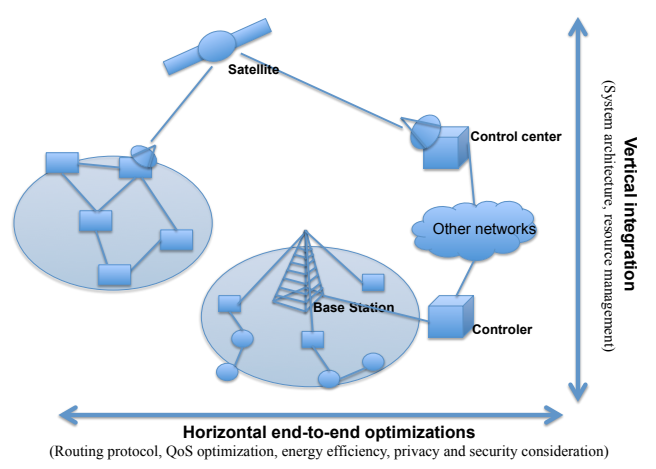

Fig. 3. Concept of integrated network and design considerations.

overall idea about MANET-Cellular mobile data offloading system is to divert some traffic in certain areas and alleviate the enormous traffic pressure making use of different networks and technologies based on their distinguished features. Taking MANET-Cellular as an entirely system, try to balance the traffic load in two networks through Wi-Fi ad-hoc technology.

\section{B. QoS optimization}

There are many different types of application data, like voice, video, web browsing, sensor data, etc. and they require different delay-sensitivities and loss-sensitivities. It is difficult to provide appropriate service levels for each kind of data to achieve satisfied system performance, which is defined as Quality of Service (QoS). QoS is essential to ensure users satisfaction in the hybrid networks where different characteristics of networks coexist and as well as various types of traffic.

In hybrid MANET-Satellite network, taking into account QoS, we can envisage two cases. The first one is QoS oriented routing protocol, where the choice of best route depends on the QoS parameters and link quality. Different metrics (e.g. delay, loss rate, jitter or throughput) can be used in path selection algorithms to achieve high level of service satisfaction under specific objectives. The second one is QoS mechanisms independent from routing protocol. The implemented QoS mechanisms can be achieved in two ways differentiated service (DiffServ) and integrated service (IntServ). DiffServ is a priority-based principle by classifying traffic flows based on their comparable characteristics. IntServ provides complete guarantee of service for each flow by reserving necessary resources all along the path of each flow. Both ways present advantages and drawbacks. IntServ provides the best level of QoS for all the flows, but it limit the available resource and introduces additional traffic by applying reservation protocol. In DiffServ, based on QoS policy for different classes, appropriate behavior will be applied by intermediate nodes. Hence, no additional traffic is introduced which makes DiffServ suitable for large network.

In MANET-cellular network, this kind of offloading approach is quite attractive since there is little or no monetary cost associated with it. However, it can be challenging to provide QoS guarantee due to a number of factors, such as heterogeneous traffic data in terms of delay and content size, various subscribing interests and limited storage of mobile users. The authors in [3] formulate the mobile data offloading problem considering heterogeneous data traffics and limited storage resources constraints. The storage allocation mechanism is designed for the end-users to maximize the system-offloading utility function. An improved greedy algorithm considers the gain per unit length, locating as many items as possible. Given the scenario of short data lifetimes, approximation algorithm is introduced. If the content sizes are identical and contact rates of mobile users are the same in the network (homogeneous scenario), system utility function can be obtained from just the popularity of data items, the lifetimes and helpers buffer sizes. Even though the problem has been formulated properly, it remains issues to be considered in realistic situation, such as when and how long the mobile users are willing to be participate in the procedure and how much space the mobiles users are willing to share.

Serving for different purposes, the optimization mechanisms of different systems have different emphasises. The studies for QoS optimization in integrated MANET-Satellite system mainly focus on the data transmission and path quality. On the contrary, in the integrated MANET-Cellular network, the studies emphasize on the characters of traffic data and mobile users. Research about how to provide QoS for different applications and dynamic situations in MANET-Satellite system has achieved some progress and that in MANET-cellular system just started. Learning from studies in MANET-Satellite network, several aspects need to be considered in MANETCellular, such as modeling about the users interesting content sizes and lifetimes, modeling about users contact and mobility, prediction about users inventive and so on. Besides, traffic classification and prioritization in this system should also be considered.

\section{Energy efficiency}

For mobile devices, keep Wi-Fi interface turned on causes significant battery drain. Previous studied have concluded that most of the power consumption on MANET nodes is spent on transmission of information. Thus, the nodes along on the shortest path or have a large number of neighbors are possibly consumed more power. In either emergency scenario or dataoffloading network, end user devices are limited in battery life and will die due to battery drained off. Hence, energy efficient mechanism is necessary to maximize the minimum lifetime of nodes and further prolong the overall network lifetime. Since the network cannot control the state of Wi-Fi interface of a user device, the energy efficiency mechanism needs to be designed in terms of reducing the transmission power.

In terms of mobile devices, energy consumption can be made based on the number of messages exchanged and their frequency. Some energy efficiency mechanisms are designed to reduce the overhead messages in order to preserve resources for data transmission [6]. Some energy aware mechanisms are based on the observation of required transmission power level at the sender and in hence estimate total transmission power of available paths [14]. Reduce of power consumption of packet retransmissions also benefits the overall energy efficiency. In addition to that, with the help of location-aware assistant, power consumption can be improved since the decrease of retransmission of packets out of reach nodes. 
TABLE II

CONSTRAINTS IN INTEGRATED SYSTEMS

\begin{tabular}{l||l|l}
\hline & MANET-Satellite & MANET-Cellular \\
\hline \hline Routing protocol & $\begin{array}{l}\text { Gateway selection; } \\
\text { Loadbalancing }\end{array}$ & $\begin{array}{l}\text { Target-set selection; Op- } \\
\text { portunistic routing }\end{array}$ \\
\hline QoS provision & $\begin{array}{l}\text { QoS mechanism; De- } \\
\text { livery reliability }\end{array}$ & $\begin{array}{l}\text { Limited storage } \\
\text { constraint; }\end{array}$ \\
\hline Energy efficiency & $\begin{array}{l}\text { Limited device bat- } \\
\text { tery; }\end{array}$ & Limited device battery; \\
\hline $\begin{array}{l}\text { Security and pri- } \\
\text { vacy }\end{array}$ & delivery reliability & $\begin{array}{l}\text { Users' interesting content; } \\
\text { Users' location informa- } \\
\text { tion; }\end{array}$ \\
\hline
\end{tabular}

In the system level, network benefits more energy efficiency from gateway/terminal. Without degrading the network performance, gateways may be turned off to preserve energy. With the assistant of gateway selection and handover mechanisms, efficient gateway activation policy can be designed and developed in the system to achieve energy efficiency.

\section{Privacy and security}

One fundamental vulnerability of MANET comes from its open peer-to-peer architecture. Unlike wired networks, any mobile node in ad hoc network can work as a router and forward packets for other nodes. The wireless channel is accessible by public and this makes devices vulnerable to malicious access. Integrating the MANET with other networks makes this stand out in some cases. In integrated MANETSatellite for emergency response system, end terminals are willing to be discovered, hence, the external access can be tolerable to certain extend. However, in cellular data offloading system, any insecure access to network information may reveal mobile users' privacy. The security should be done properly in this system. Firstly, with regard to the procedure, the users should have the absolutely right to be participate in and out of the process anytime. In paper [12], the authors also considered the willingness of the users. To start this data offloading procedure, it is the end users who decide whether or not to share the information with others and when to be in and out of the process. Network providers can only choose the users those are willing to participate in the information dissemination process. Secondly, with regard to the content, the user privacy needs to be protected in terms of several aspects, such as content information which leads to the users' interests and location information which reveals the movement and activity of users. In project Serval [9], voice calls and text messages of the mobile users are encrypted to support security.

\section{CONCLUSION}

The most useful feature of MANET is spontaneous communication anywhere and anytime (within communication range). When integrated MANET with other networks and designed for different purposes, the highlight and challenges will be different. In this paper, we examine features of the two integrated systems. The studies about addressing problems and enhancing network performance are also presented. Comparisons of the two systems in terms of network characteristics and realistic studies are significant for their further studies and development. For emergency services, ubiquitous and robust communications are of paramount importance. During the emergency response operations, most of the data traffic are of small size but crucial. Delivery of this kind of traffic requires high reliability and minimum delay. QoS guarantee has higher priority than the storage and energy concern. Hence, the centre of the studies in MANET-Satellite network is about reliable transmission and successful delivery. For the mobile data offloading services, the network faces enormous and various types of data traffic. Design in this case focuses on the mobile users, such as storage allocation, energy efficient and privacy protection. Table II presents comparisons of the main constraints considered in each aspect in integrated networks. Studies about integrated MANET-Satellite network for emergency services have made progress and exploring in MANETCellular data offloading network just starts to flourish. Hence, the further research can benefit from taking advantage of the similarity of two systems and tackling the discrepancy. For example, some of the routing protocols proposed in MANETSatellite system can be improved and applied in MAENTCellular system. To achieve full-scale QoS provisioning in MANET-Cellular network, it is efficient to build specific algorithms on top of the QoS mechanisms already proposed in MANET-Satellite networks. With the common constraints for energy efficiency, the same algorithms can be used in both systems to deal with the mutual problem.

\section{REFERENCES}

[1] T. Taleb, Y. H. Aoul, and T. Ahmed, "Challenges, opportunities, and solutions for converged satellite and terrestrial networks." IEEE Wireless Commun., vol. 18, pp. 46-52, 2011.

[2] A. Oliveira, Z. Sun, M. Monier, P. Boutry, and D. Gimenez, "On optimizing hybrid ad-hoc and satellite networks the monet approach," in Proceedings of Future Network and Mobile Summit Conference, 2010.

[3] Y. Li, G. Su, P. Hui, D. Jin, L. Su, and L. Zeng, "Multiple mobile data offloading through delay tolerant networks," in Proceedings of the 6th ACM Workshop on Challenged Networks, ser. CHANTS '11. ACM, 2011, pp. 43-48.

[4] A. Aijaz, H. Aghvami, and M. Amani, "A survey on mobile data offloading: technical and business perspectives." IEEE Wireless Commun., vol. 20, pp. 104-112, 2013.

[5] E. Re, S. Jayousi, S. Morosi, and L. Ronga, "Salice project: Satelliteassisted localization and communication systems for emergency services," IEEE Aerospace and Electronic Systems Magazine, vol. 28, pp. 2-13, 2013.

[6] C. Michael, "Algorithms and mechanisms for system optimization," MONET project report, 2012.

[7] "The dumbo project: Digital ubiquitous mobile broadband olsr," http://www.ait.ac.th/news-and-events/archive/2006/, accessed: 2013-1230 .

[8] B. Evans, "Integration of satellite and terrestrial systems in future multimedia communications," IEEE Wireless Communication, vol. 12, 2005.

[9] "The serval project: Practical wireless as-hoc mobile telecommunications," http://servalproject.org/, accessed: 2013-12-30.

[10] "Dythr-local headline dissemination," http://seym.net/dythr/, accessed: 2013-12-30.

[11] V. Vukadinovic and G. Karlsson, "Spectrum and energy efficiency of mobility-assisted podcasting in cellular networks," in MobiOpp '10 Proceedings of the Second International Workshop on Mobile Opportunistic Networking, 2010, pp. 51-57.

[12] B. Han, P. Hui, V. S. A. Kumar, M. V. Marathe, J. Shao, and A. Srinivasan, "Mobile data offloading through opportunistic communications and social participation," IEEE Transactions on Mobile Computing, vol. 11, pp. 821-834, 2012.

[13] H. Izumikawa and J. Katto, "Rocnet: Spatial mobile data offload with user-behavior prediction through delay tolerant networks." in WCNC. IEEE, 2013.

[14] J. Zhu and X. Wang, "Model and protocol for energy-efficient routing over mobile ad hoc networks," IEEE Transactions on Mobile Computing, vol. 10, pp. 1546-1557, 2011. 